\title{
CHARACTERIZATION OF POROUS MEDIA BY A SIZE SENSITIVE HOMOGENIZATION APPROACH
}

\author{
LUDMILA PARASHKEVOVA* \\ Institute of Mechanics, Bulgarian Academy of Sciences, \\ Acad. G. Bonchev. Str., Bl. 4, 1113 Sofia, Bulgaria \\ [Received: 03 April 2020. Accepted: 05 October 2020] \\ doi: 10.7546/JTAM.50.20.04.04
}

\begin{abstract}
The present study concerns the mechanical properties assessment of porous media with a moderate porosity of a closed-cells type. On the base of the generalized mixture rule coupled with a size-sensitive variant of MoriTanaka theory the new explicit formulae are obtained. The elastic characteristics and the initial yield stress of a porous composite are calculated by two models, accounting for pore sizes. In both model variants the matrix is considered as a micropolar centrosymmetric medium. The size effect predicted depends on the ratio between the mean pore size/ the matrix intrinsic length. It is stronger if the pores are very small, but always remains limited.
\end{abstract}

KEY WORDS: closed-cell foam, homogenization, pore size effect.

\section{INTRODUCTION}

Accounting for the influence of sizes on the properties of porous composites is carried out in two different aspects. The first aspect is related to the influence of the size of porous samples during registration of material characteristics, and the second one - to the study of the influence of the pores size in the representative volume of the porous composites and pores size distribution on thermo-mechanical and other physical properties [1-4].

Essentially, this study is a continuation of the work [5] aimed to develop models capable to take into account the size effects of the second type. For this purpose, the phenomenological approach of Generalized Mixture Rule (GMR) [6,7], which is well established for the description of the elastic moduli of porous materials of different nature is associated with the size-sensitive version of Mori-Tanaka method $[8,9]$. Two homogenization models are obtained and they give vastly different estimates of the studied moduli. Since the presented models have a clear analytical description, it is easy to compare them with the models suggested by other authors, for example, those presented in review articles [10,11].

\footnotetext{
${ }^{*}$ Corresponding author e-mail: lusy@imbm.bas.bg
} 


\section{Modelling}

A closed-cells porous material is considered as a two-phase composite based on a micropolar centrosymmetric matrix, containing randomly distributed spherical inclusions (pores) with average diameter $D_{i}$. In this section two size-sensitive homogenization models SSGMR1 and SSGMR2, based on GMR will be presented. After homogenisation procedures the overall material is supposed to be a classic isotropic Cauchy one.

\subsection{Elastic STATE}

The following elastic constitutive equations are valid for the matrix [12]:

$$
\begin{array}{lll}
\sigma_{(i j)}^{\prime}=2 G_{m} \varepsilon^{\prime}{ }_{(i j)} & \sigma_{<i j>}=2 \varkappa \varepsilon_{<i j>} & \sigma_{(k k)}=3 K_{m} \varepsilon_{(k k)} \\
m_{(i j)}^{\prime}=2 \beta k_{(i j)}^{\prime} & m_{<i j>}=2 \gamma k_{<i j>} & m_{(k k)}=3 N k_{(k k)} .
\end{array}
$$

The stress and strain measures are $\sigma_{i j}=\sigma_{(i j)}+\sigma_{<i j>} ; \varepsilon_{i j}=\varepsilon_{(i j)}+\varepsilon_{<i j>}$, couple stress and curvature tensor $m_{i j}=m_{(i j)}+m_{<i j>} ; k_{i j}=k_{(i j)}+k_{<i j>}$, respectively. The symbol $\{\ldots\}_{i j}^{\prime}$ is a notation for a tensor deviator; (...) and $\langle\ldots\rangle$ in the subscripts denote symmetric and anti-symmetric parts of a tensor. Cauchy and Cosserat constants of the matrix are related by the following equations [9]:

$$
l_{m}^{2}=\beta / G_{m}=\gamma / \varkappa, \quad \varkappa / G_{m}=\gamma / \beta=p .
$$

If $p=1$ one gets a simpler variant of the theory, where:

$$
\varkappa=G_{m}, \quad \gamma=\beta=l_{m}^{2} G_{m}, \quad N=l_{m}^{2} K_{m},
$$

$l_{m}$ is an internal length of the matrix and the index $m$ is set for the matrix properties. The basic idea of the GMR is expressed by the assumption that a particular overall property $M_{c}$ of the two-phase porous composite can be presented by the power law

$$
M_{c}{ }^{J}=(1-c) M_{m}{ }^{J},
$$

where $J$ is a model parameter, which depends on matrix, composite microstructure and on other manufacturing conditions. Usually the parameter $J$ is experimentally fitted. Our approach presumes this parameter to be linked to the size-sensitive variant of Mori-Tanaka theory (SSMT) [9] for the bulk and shear moduli, respectively

$$
\begin{aligned}
K_{\text {SSMT }} & =K_{m}-\frac{K_{m} C}{1-\frac{3(1-C)}{3+4 G_{m} / K_{m}}}, \\
G_{\text {SSMT }} & =G_{m}-\frac{G_{m} C}{1-(1-C)\left(b_{m}-b_{0 i}\right)}, \quad b_{m}=\frac{6\left(K_{m}+2 G_{m}\right)}{5\left(3 K_{m}+4 G_{m}\right)} .
\end{aligned}
$$


The size-sensitive parameter $b_{0 i}$ depends on the micropolar properties of the matrix material and on the pore diameter $D_{i}$ :

$$
\begin{aligned}
& b_{0 i}=\frac{6 p}{5(p+1)} R_{i}\left(\eta_{i}\right), \\
& R_{i}\left(\eta_{i}\right)=e^{-\eta_{i}}\left(\eta_{i}^{-2}+\eta_{i}^{-3}\right)\left(\eta_{i} \cosh \eta_{i}-\sinh \eta_{i}\right), \\
& \eta_{i}=\frac{\sqrt{p}}{(p+1)} \frac{D_{i}}{l_{m}} .
\end{aligned}
$$

In general, $0 \leq b_{0 i} \leq 2 / 5$.

Following the condition:

$$
K_{c}^{\prime}(0)=K_{S S M T}^{\prime}(0), \quad G_{c}^{\prime}(0)=G_{S S M T}^{\prime}(0)
$$

( ( ... $)^{\prime}$ means a derivative with respect to porosity $C$ ) one gets for the model variant SSGMR1:

$$
\begin{gathered}
\alpha_{K(\text { SSGMR } 1)}=\alpha_{K}{ }^{(1)}=3\left(1-\nu_{m}\right) /\left\{2\left(1-2 \nu_{m}\right)\right\} \\
\alpha_{G(\text { SSGMR } 1)}=\alpha_{G}^{(1)}=15\left(1-\nu_{m}\right) /\left\{\left(7-5 \nu_{m}\right)+15\left(1-\nu_{m}\right) b_{0 i}\right\} ; \\
K_{c}^{(1)}=K_{m}(1-C)^{\alpha_{K}(1)} \\
G_{c}^{(1)}\left(D_{i}\right)=G_{m}(1-C)^{\alpha_{G}{ }^{(1)}} .
\end{gathered}
$$

The model parameters for the variants SSGMR2 are obtained supposing that:

$$
\alpha_{G(\text { SSGMR2 })}=\alpha_{G}{ }^{(2)}=\alpha_{G}^{(1)} .
$$

On the other hand, if we simplify the classical dependence of Poisson's ratio on bulk and shear moduli $\nu_{c}^{(2)}=\nu_{c}^{(2)}\left[K_{c}^{(2)}, G_{c}^{(2)}\right]$ through the linear approximation about porosity up to $C \rightarrow 1$ and supposing that $\alpha_{K \text { (SSGMR2) }}-\alpha_{G \text { (SSGMR2) }}>0$, then $\nu_{c}^{(2)}(C=1)=-1$ and

$$
\begin{gathered}
\alpha_{G}^{(2)}=15\left(1-\nu_{m}\right) /\left\{\left(7-5 \nu_{m}\right)+15\left(1-\nu_{m}\right) b_{0 i}\right\} \\
\alpha_{K}^{(2)}=3 /\left(1-2 \nu_{m}\right)+15\left(1-\nu_{m}\right) /\left\{\left(7-5 \nu_{m}\right)+15\left(1-\nu_{m}\right) b_{0 i}\right\} ; \\
K_{c}^{(2)}\left(D_{i}\right)=K_{m}(1-C)^{\alpha_{K}^{(2)}} \\
G_{c}^{(2)}\left(D_{i}\right)=G_{m}(1-C)^{\alpha_{G}^{(2)}} .
\end{gathered}
$$

The dependence of parameters $\alpha_{G}^{(1)}, \alpha_{K}^{(1)}$ and $\alpha_{G}^{(2)}, \alpha_{K}{ }^{(2)}$ on matrix Poisson's ratio at different values of $b_{0 i}$ are illustrated on Fig. 1: It is interesting to note that 


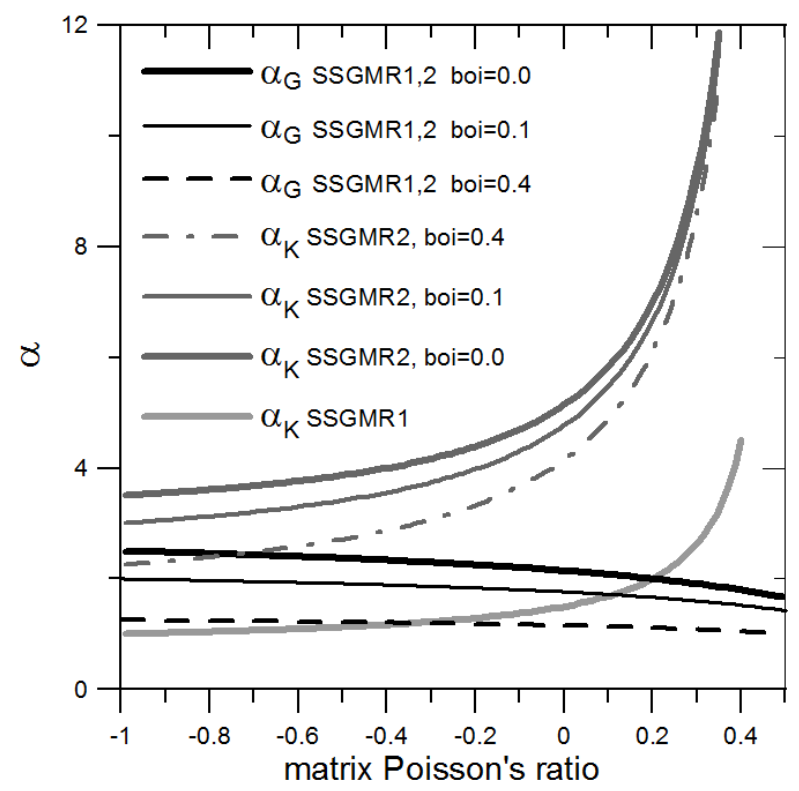

Fig. 1: Influence of matrix Poisson's ratio on the exponents for the models SSGMR1 and SSGMR2.

the other two important overall elastic constants - Young's modulus and Poisson's ratio - depend on the difference $\alpha_{K}{ }^{(i)}-\alpha_{G}{ }^{(i)}, i=1,2$, which is for different model variants, respectively:

$$
\begin{aligned}
& \alpha_{K}{ }^{(1)}-\alpha_{G}{ }^{(1)}<0,-1<\nu_{m}<\nu_{m}{ }^{*}, \\
& \alpha_{K}{ }^{(1)}-\alpha_{G}{ }^{(1)}=0, \nu_{m}=\nu_{m}{ }^{*}, \\
& \alpha_{K}{ }^{(1)}-\alpha_{G}{ }^{(1)}>0, \nu_{m}{ }^{*}<\nu_{m}<1 / 2, \\
& \nu_{m}{ }^{*}=\frac{1-5 b_{0 i}}{5\left(1-b_{0 i}\right)},-1 / 3 \leq \nu_{m}{ }^{*} \leq 1 / 5, \\
& \alpha_{K}{ }^{(2)}\left(D_{i}\right)-\alpha_{G}{ }^{(2)}\left(D_{i}\right)=\frac{3}{1-2 \nu_{m}}>0 .
\end{aligned}
$$

In Fig. 2 and Fig. 3 the behaviour of the relative overall Young's modulus according to models SSGMR1 and SSGMR2 at porosity changes is plotted for two matrix materials. A matrix with Poisson's ratio $\nu_{m}=0.14$ (like $\mathrm{SiC}$ ) belongs to the group of brittle materials, see Fig. 2, whereas a martix from Fig. 3 with Poisson's ratio $\nu_{m}=0.4$ (like Pd alloy) usually demonstrates high ductility.

Existing models for homogenization of porous media, describing changes in the stress-measured main elastic moduli, give more or less similar descending curves 


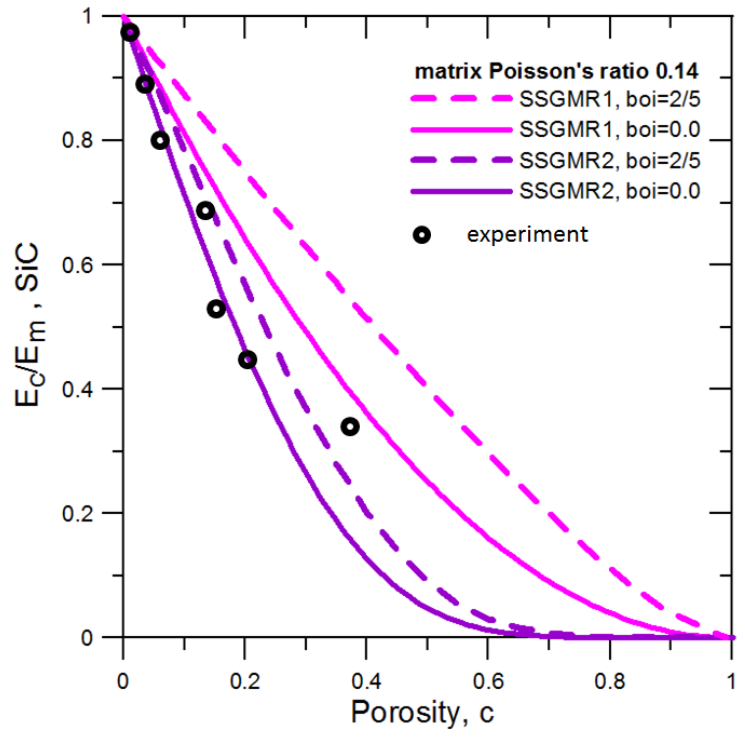

Fig. 2: Dependence of the relative Young's modulus on porosity, $\nu_{m}=0.14$. Experimental data on porous $\mathrm{SiC}$ [13].

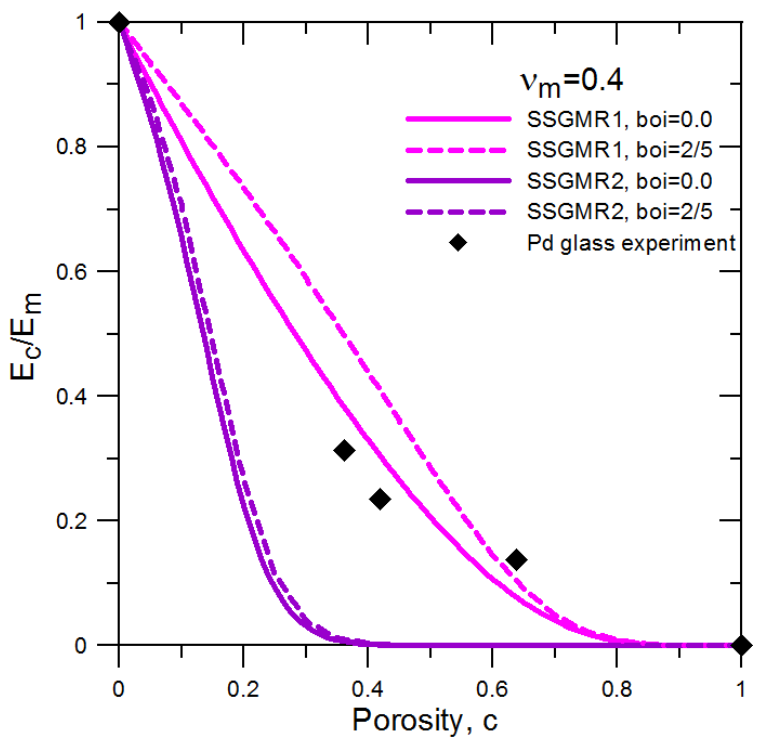

Fig. 3: Dependence of the relative Young's modulus on porosity, $\nu_{m}=0.4$. Experimental data on porous Pd glass [14]. 


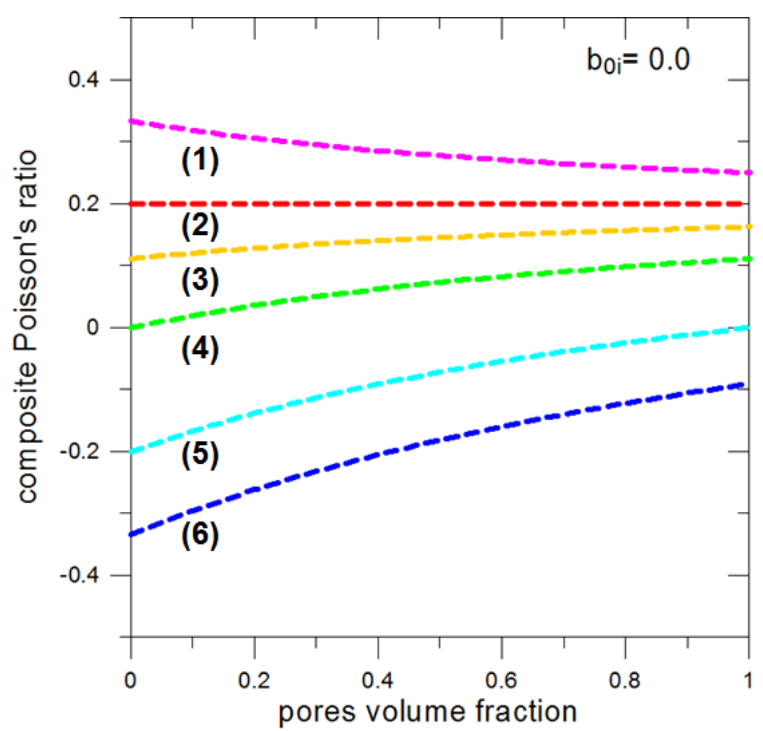

Fig. 4: Dependence of Poisson's ratio on porosity, according to Mori-Tanaka theory, $\left(\mathrm{SSMT}, b_{0 i}=0\right)$.

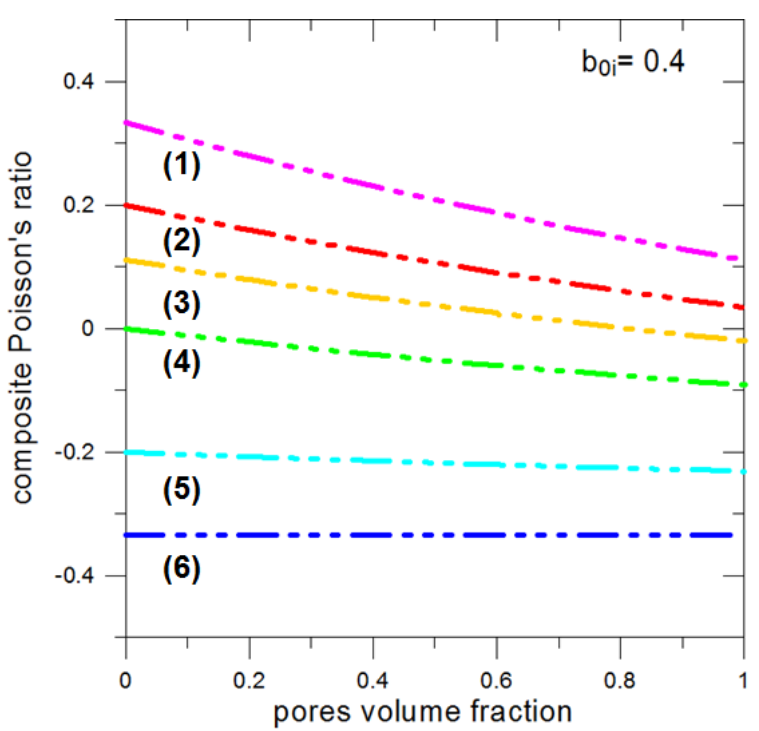

Fig. 5: Dependence of Poisson's ratio on porosity, according to size-sensitive MoriTanaka theory [9], (SSMT, $\left.b_{0 i}=2 / 5\right)$. 


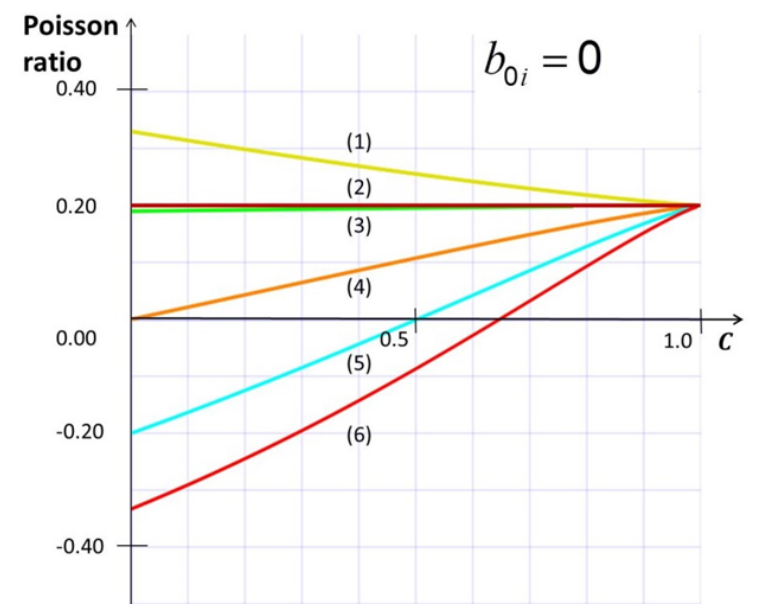

Fig. 6: Dependence of Poisson's ratio on porosity, according to differential MoriTanaka theory, (SSDMT, $b_{0 i}=0$ ).

with increasing porosity, for example see $[11,15]$. These curves are determined by the initial matrix moduli and by zero values at high porosity up to unity. However, the situation with the prediction of the behaviour of Poisson's ratio with increasing porosity, see [16], is still very ambiguous, especially in the absence of sufficiently experimental data.

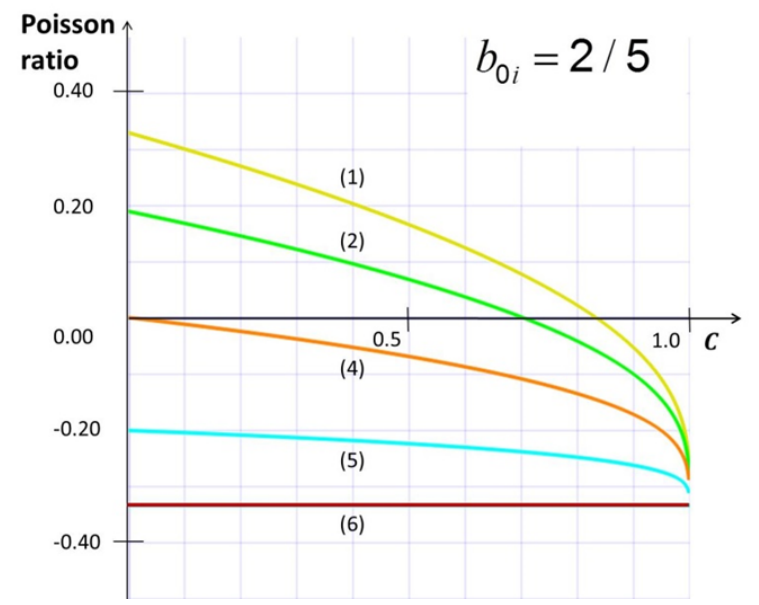

Fig. 7: Dependence of Poisson's ratio on porosity, according to size-sensitive differential Mori-Tanaka theory [17] (SSDMT, $b_{0 i}=2 / 5$ ). 


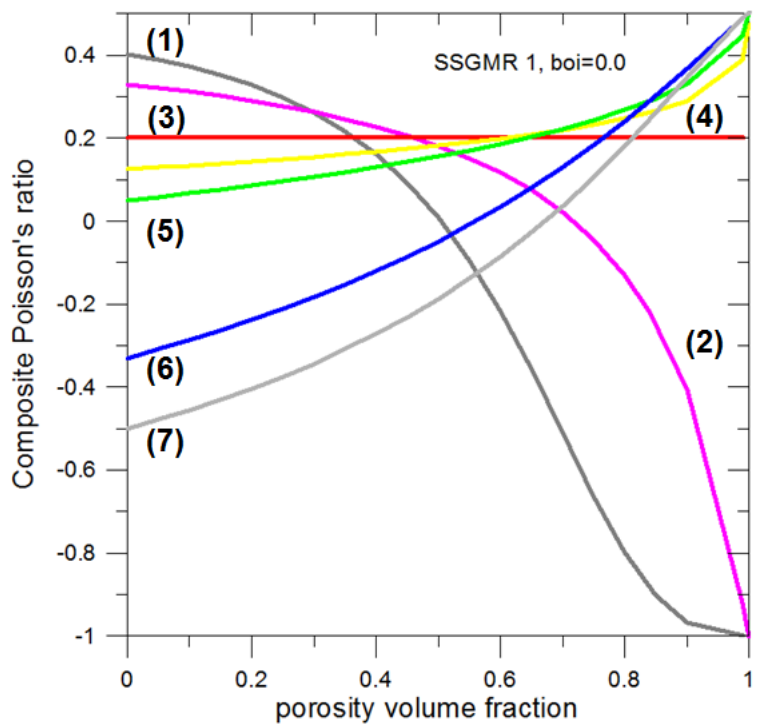

Fig. 8: Dependence of Poisson's ratio on porosity, according to the present model SSGMR1 at $b_{0 i}=0$.

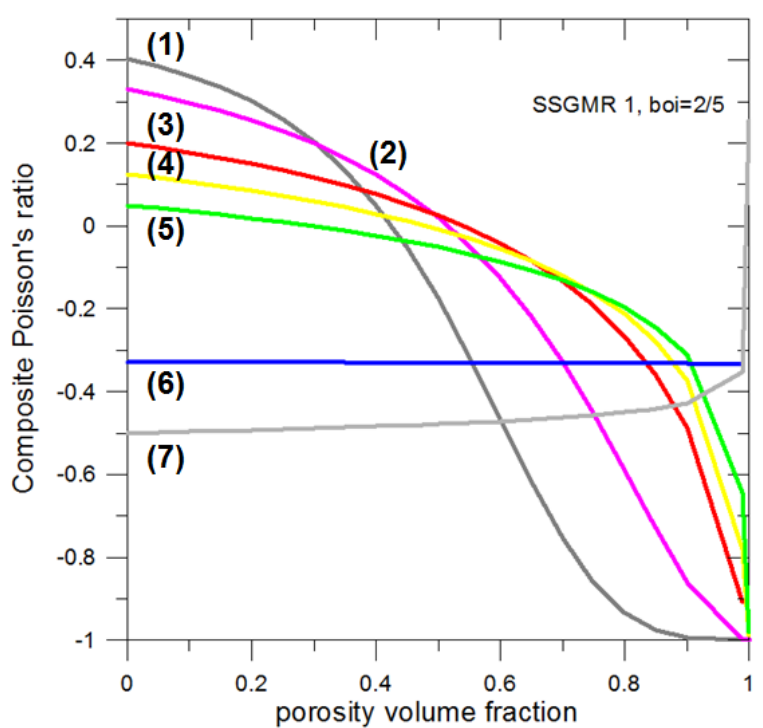

Fig. 9: Dependence of Poisson's ratio on porosity, according to the present model SSGMR 1 at $b_{0 i}=2 / 5$. 


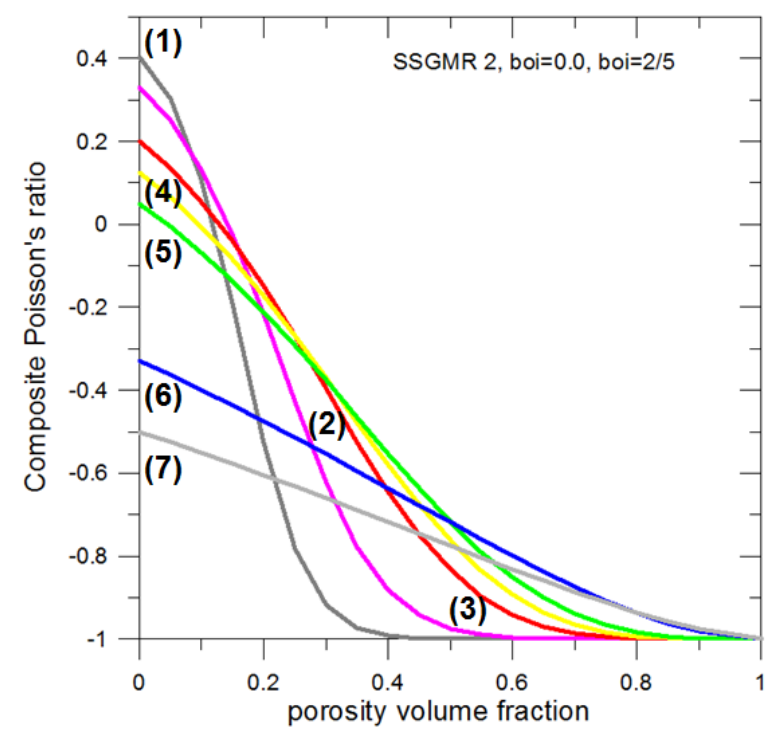

Fig. 10: Dependence of Poisson's ratio on porosity, according to the present model SSGMR2.

To illustrate this variation, predictions of Poisson's ratio are presented for 6 different theories. Figure 4 shows the change in Poisson's ratio according to the classical Mori-Tanaka model, Fig. 5 - according to size-sensitive Mori-Tanaka model (SSMT), Fig. 6 and Fig. 7 represent differential Mori-Tanaka and differential size-sensitive Mori-Tanaka model (SSDMT) [17], respectively. Curves obtained from SSGMR1 model are shown in Fig. 8 and Fig. 9. For clarity, only the limiting cases $b_{0 i}=0$ (no size effect), and $b_{0 i}=2 / 5$ (maximal size effect) are demonstrated. Finally the data linked to SSGMR2 are plotted in Fig. 10. In this variant Poisson's ratio does not depend on $b_{0 i}$, see (11). On all cited figures the curves (1)-(6), (7) correspond to composites with different matrices and have been numbered following decreasing value of the initial Poisson's ratio.

\subsection{INITIAL PLASTIC STATE}

At the present stage of research, we do not have a goal to derive a model describing the whole complex process of deformation of porous materials beyond elasticity. We restrict ourselves to determining the conditions for the onset of inelastic flow of a composite in terms of macro-stresses.

So, it is accepted that the non-elastic state of the porous composite will occur when a certain equivalent stress averaged over the matrix volume in the RVE reaches 
the yield strength of the matrix. Next, we follow the deriving procedure for the plasticity condition presented in [5], but with an accent on the fact that in our case the matrix is a material of Cosserat type.

Thus, the obtained new plasticity conditions repeat the previously derived equation in form and differ significantly in the values of parameters $\boldsymbol{A}$ and $\boldsymbol{H}$. The current conditions depend not only on the concentration, but also on the size of spherical pores. $A_{(i)}\left(D_{i}\right)$ and $H_{(i)}\left(D_{i}\right), i=1,2$ denote the parameters of corresponding yield condition for SSGMR1 and SSGMR2:

$$
\frac{3}{2} S_{i j} S_{i j}+\boldsymbol{A}_{(i)}^{2} \frac{\Sigma_{k k}{ }^{2}}{9}=\boldsymbol{H}_{(i)}^{2} \sigma_{p m}{ }^{2},
$$

where

$$
\begin{aligned}
\boldsymbol{A}_{(i)}^{2}= & \frac{\left[(1-C)-\frac{K_{c}{ }^{(i)}}{K_{m}}\right]}{\left[(1-C)-\frac{2}{3} \frac{G_{c}^{(i)}}{K_{m}}\right]} \frac{G_{c}^{(i)^{2}}}{K_{c}^{(i)^{2}}} ; \\
\boldsymbol{H}_{(i)}^{2}= & \frac{(1-C)\left[1-\frac{2}{3} \frac{G_{m}}{K_{m}}\right]}{\left[(1-C)-\frac{2}{3} \frac{G_{c}{ }^{(i)}}{K_{m}}\right]} \frac{G_{c}^{(i)^{2}}}{G_{m}^{2}} ; \quad i=1,2 .
\end{aligned}
$$

The conditions (15) are applicable, if $\nu_{m}>0$. Substituting the uniaxial compression/ tension macro-stress state, relation (15) can be used for prediction of the corresponding yielding point $\sigma^{1}{ }_{p c(i)}$ :

$$
\frac{\sigma_{p c(i)}^{1}}{\sigma_{p m}}=\frac{3 \boldsymbol{H}_{(i)}\left(\nu_{m}, b_{0 i}, C\right)}{\sqrt{9+\boldsymbol{A}_{(i)}{ }^{2}\left(\nu_{m}, b_{0 i}, C\right)}} .
$$

\section{Numerical Simulations}

In this section some results will be discussed on the view point of the practice applicability of the model variants suggested. As it was shown in section 2 the estimates given by the model SSGMR2 are much lower than those obtained by means of the model SSGMR1. Therefore, Fig. 11 represents the results for the Young's modulus associated only to the model SSGMR1, SSDMT model [17] and some analytical models of other authors obtained at different times, especially Archie's moodel [20], Gibson-Ashby's model [21], one of Pabst-Gregorova's variants [15] 


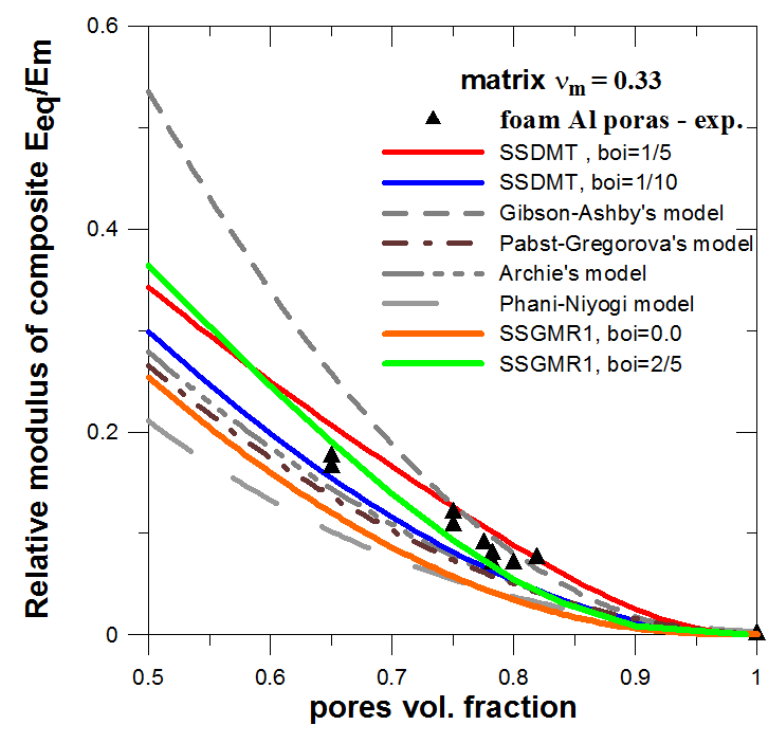

Fig. 11: Dependence of the relative Young's modulus on porosity, $\nu_{m}=0.33$, corresponding to SSGMR1, other analytical models and experiments on Alporas foam [11].

and Phani-Niyogi's model [22]. The experiments carried out by [23] for one of the most popular foams based on aluminium - Alporas -are shown also on the same figure. Together with other comparisons (for porous palladium alloy [14], see Fig. 3; for a porous material based on titanium [18], see Fig. 12 and for a nickel foam [19], see Fig. 13) these results give reason to argue that the model SSGMR1 is comparable to other well-known models, and predicts acceptable estimates of elastic properties over a wide range of porosity.

Regarding Poisson's ratio for closed cell porous materials, there exist a few experiments published, which cover more or less wide porosity interval. With this background, we chose to illustrate the possibilities of the model SSGRM1 by comparing our data with experiments on porous uranium oxide $\left(\mathrm{UO}_{2}, \nu_{m}=0.32\right)$, which show a downward curve with an increase in porosity [24], see Fig. 14, and comparisons with experiments on porous sand $\left(\mathrm{SiO}_{2}, \nu_{m}=0.159\right)$ from [7], where a tendency for a slight increase of Poisson's ratio is reported, see Fig. 15. 


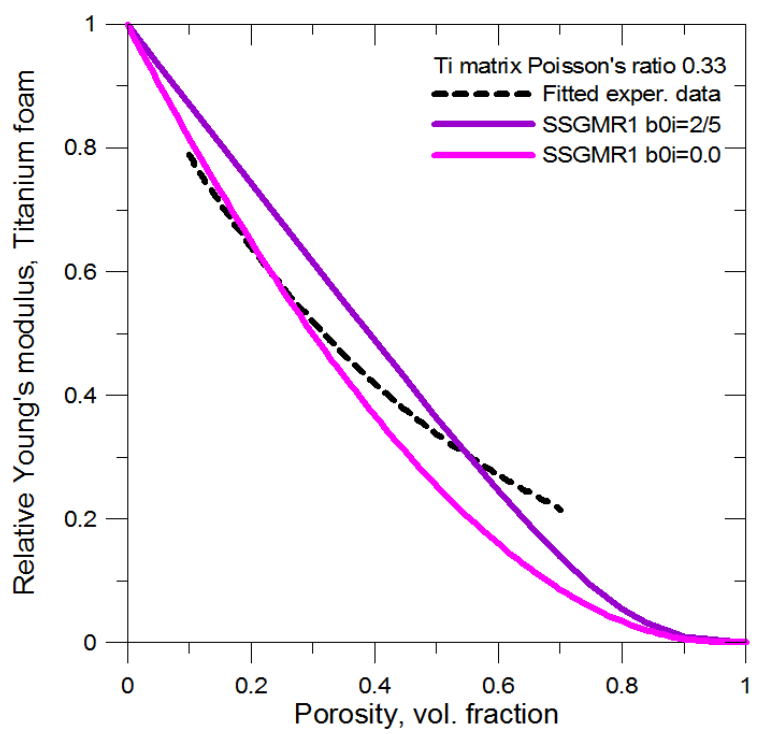

Fig. 12: Relative Young's modulus v/s porosity. Comparison among limiting cases of SSGMR1 and experiments on Ti-based foam [18].

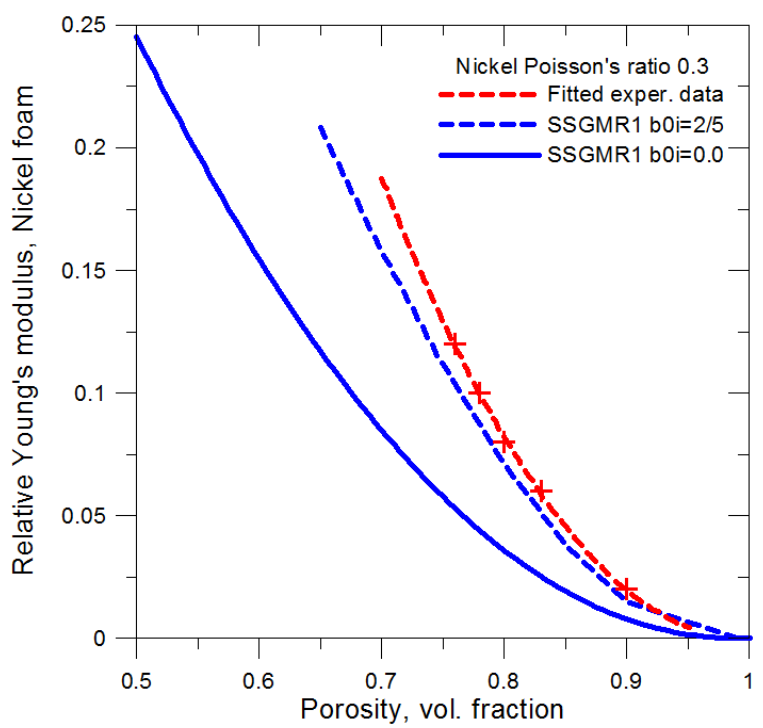

Fig. 13: Relative Young's modulus vs. porosity. Comparison among limiting cases of SSGMR1 and experiments on Ni-based foam [19]. 


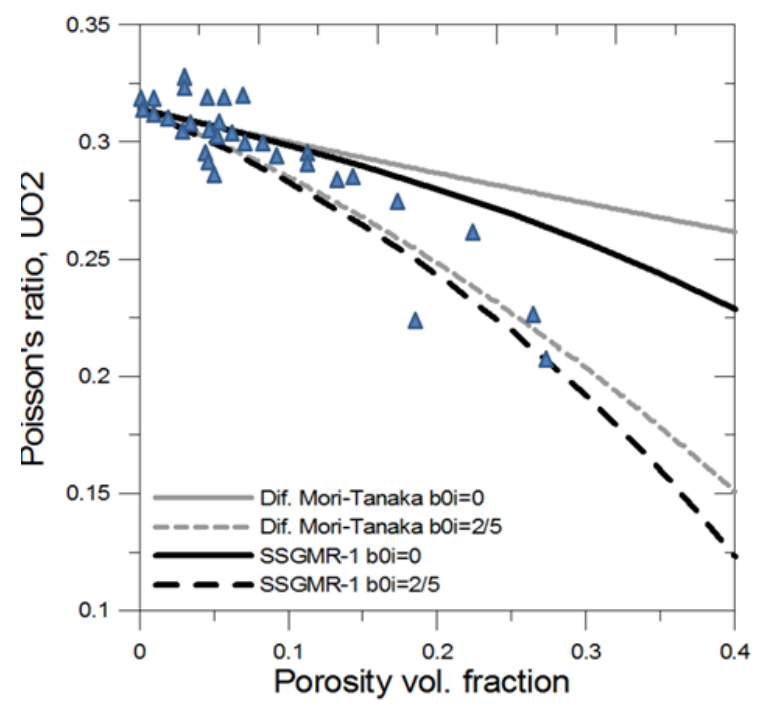

Fig. 14: Poisson's ratio vs. porosity, $\nu_{m}=0.32$. Comparison among limiting cases of SSGMR1 and experiments on porous uranium dioxide [22].

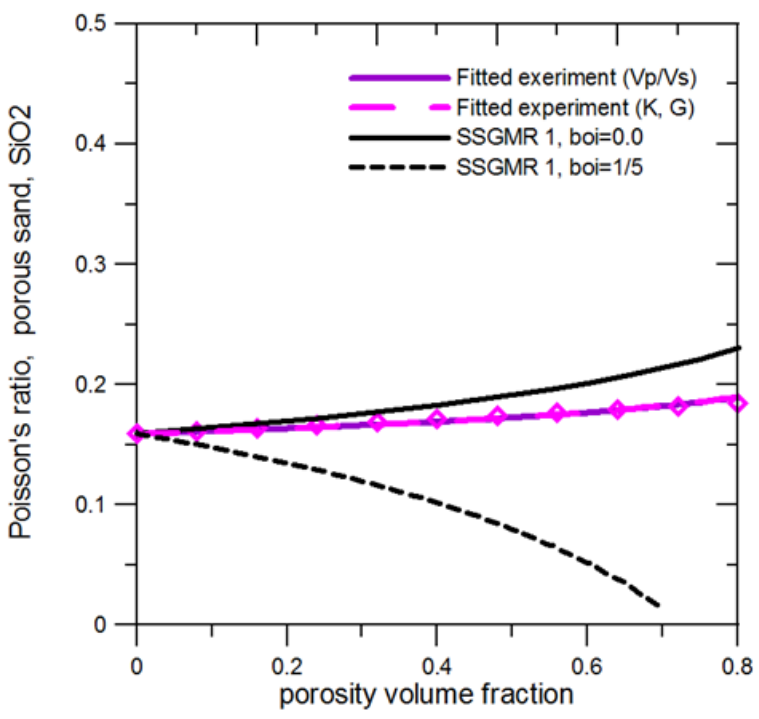

Fig. 15: Poisson's ratio vs. porosity, $\nu_{m}=0.159$. Comparison among limiting cases of SSGMR1 and experiments on porous sand $\left(\mathrm{SiO}_{2}\right),[6,7]$. 


\section{Conclusions}

The juxtaposition between the theoretical data and the experiments shown on the figures from Sections 2, 3 confirms the previously noted tendency [15], that it is very difficult to cover the entire porosity change interval with one model, especially when varying the properties of the matrix over a wide range.

Based on the foregoing, we assume that SSGMR1 model is more appropriate for metal-based composites, and SSGMR2 model is more suitable for materials with lower Poisson's ratio . It should be noted that about $80 \%$ of the experimental data known to us which has been obtained from a wide variety of porous media fall between the predictions from SSGMR1 and SSGMR2. The correct analytical description of Poissons ratio behaviour for porous composites is still the most challenging task.

In the case of a two-phase porous composite due to the fact that commonly accepted lower theoretical bounds for the bulk and shear moduli are simply zero, model SSGMR2 can be used as a kind of a 'lower' bound for those properties. At the very least, as far as we know, the estimates associated to the model SSGMR2 are the lowest of all known in the literature.

\section{ACKNOWLEDGEMENTS}

The support from BG FSI through the grant No KПI-06-27/7-2018 "Experimental study and modeling of structure and mechanical behavior of high-performance fiberreinforced silicate composites" is gratefully acknowledged.

\section{REFERENCES}

[1] M. Deverge, L. Benyahia, S. Sahraoui (2009) Experimental investigation on pore size effect on the linear viscoelastic properties of acoustic foams. Journal of the Acoustical Society of America 126(3) EL 93-96.

[2] X.C. Xia, X.W. Chen, Z. Zhang, X. Chen, W.M. Zhao, B. Liao, B. Hur (2013) Effects of porosity and pore size on the compressive properties of closed-cell Mg alloy foam. Journal of Magnesium and Alloys 1(4) 330-335.

[3] Z.G. XU, J.W. FU, T.J. LuO, Y.S. YANG (2012) Effects of cell size on quasi-static compressive properties of Mg alloy foams. Mater Design 34 40-44.

[4] Takashi Uneyama, Tatsuya Yamazaki, Toshio Igarashi, Koh-hei Nitta (2019) Effect of pore size distribution on compressive behavior of moderately expanded low-density polyethylene foams. Polymer Engineering and Science 59(3) 510-518.

[5] L. Parashkevova (2020) Characterization of porous materials by homogenization models based on a generalized mixture rule. Computing in Industrial Mathematics, BGSIAM 2019, Studies in Computational Intelligence (submitted).

[6] S. JI, QI GU, B. XIA (2006) Porosity dependence of mechanical properties of solid materials. Journal of Materials Science 4117571768. 
[7] CH. YU, SH. Ji, QI Li (2016) Effects of porosity on seismic velocities, elastic moduli and Poisson's ratios of solid materials and rocks. Journal of Rock Mechanics and Geotechnical Engineering 83549.

[8] G. HU, X. LIU, T.J. LU (2005) A variational method for non-linear micropolar composites. Journal of Alloys and Compounds 37 407-425.

[9] L. Parashievova, N. Bontcheva, V. Babakov (2010) Modelling of size effects on strengthening of multiphase $\mathrm{Al}$ based composites. Computational Materials Science 50 527-537.

[10] M. Kaczmarek, M. Goueygou (2006) Dependence of elastic properties of materials on their porosity: review of models. Journal of Porous Media 9(4) 335-355.

[11] P. Koudelka, O. Jirousek, T. Doktor, P. Zlamal, T. Fila (2012) Comparative study on numerical and analytical assessment of elastic properties of metal foams. In: Proceedings of the 18th International Conference "Engineering Mechanics", May 1417, 2012, Svratka, Czech Republic, Paper no 218, pp. 691-701.

[12] W. NOWACKI (1986) "Theory of asymmetric elasticity". Pergamon Press.

[13] J.A. COppola, R.C. BRAdT (1972) Measurement of Fracture Surface Energy of SiC. Journal of the American Ceramic Society 55(9) 455-460.

[14] T. WAdA, A. InOUe (2004) Formation of porous Pd-based bulk glassy alloys by a hydrogen pressure melting - water quenching method and their mechanical properties. Mater Transactions 45(8) 2761-2765.

[15] W. Pabst, E. Gregorova (2004) New relation for the porosity dependence of the effective tensile modulus of brittle materials. Journal of Materials Science 393501 35031.

[16] J. Kovacik, L. Marsavina, E. Linul (2018) Poisson's ratio of closed-cell aluminium foams. Materials (Basel) 11(10) E1904.

[17] L. Parashkevova, L. Drenchev, P. Egizabal (2020) Mechanical properties assessments for materials of high porosity and light alloys with predominant embedded phases. Computing in Industrial Mathematics, BGSIAM 2018, Studies in Computational Intelligence (in print).

[18] S. Lascano, C. Arévalo, I. Montealegre-Melendez, S. Muñoz, J.A. Rodriguez-Ortiz, P. Trueba, Y. Torres (2019) Porous titanium for biomedical applications: evaluation of the conventional powder metallurgy frontier and spaceholder technique. Applied Sciences 9(5) 982.

[19] T. Dillard (2004) Caracterisation et simulation numerique du comportement mecanique des mousses de nickel: morphologie tridimensionnelle, reponse elastoplastique et rupture. PhD Thesis, Ecole des mines de Paris.

[20] G.E. ARCHIE (1942) The electrical resistivity log as an aid in determining some reservoir characteristics. Transactions of the American Institute of Mining, Metallurgical, and Petroleum Engineers 146 54-67.

[21] M.F. Ashby, A.G. Evans, N.A. Fleck, L.J. Gibson, J.W. Hutchinson, H.N.G. WADlEY (2000) "Metal Foams: A Design Guide", Butterworth-Heinemann, Boston, MA, USA, $43 \mathrm{p}$. 
[22] K.K. Phani, S.K. NiYOgi (1987) Young's modulus of porous brittle solids. Journal of Materials Science 22 257-263.

[23] J. KovaciK, F. SimanciK (1998) Aluminium foam - Modulus of elasticity and electrical conductivity according to percolation theory. Scripta Mater 39 239-246.

[24] J.P. PANAKKAL (1991) Use of longitudinal ultrasonic velocity as a predictor of elastic moduli and density of sintered uranium dioxide. IEEE Transactions on Ultrasonics, Ferroelectrics, and Frequency Control 38(3) 161-165. 